\title{
Treino Cognitivo e Intervenção Psicoeducativa para Indivíduos Hipertensos: Efeitos na Cognição
}

\author{
Cognitive Training and Educational Intervention for Individuals \\ with Hypertension: Effects on Cognition
}

\author{
Thais Bento Lima-Silva \& Monica Sanches Yassuda* \\ Universidade de São Paulo, São Paulo, Brasil
}

\begin{abstract}
Resumo
Objetivos: Avaliar a possibilidade de aliar o treino cognitivo à intervenção psicoeducativa de oito sessões sobre hipertensão visando melhor manejo desta condição crônica em idosos. Métodos: Participaram 64 idosos que se declararam como hipertensos, divididos em grupo experimental (GE, $n=35$ ) e grupo controle (GC, $n=29)$. O grupo controle recebeu treino após o pós teste. O protocolo incluiu dados sócio-demográficos e clínicos, o Mini- Exame do Estado Mental (MEEM), Escala de Depressão Geriátrica (EDG), Teste Comportamental de Memória de Rivermead (RBMT), Fluência Verbal categoria animais (FV) e Short Cognitive Test (SKT). Resultados: Pôde-se observar que o GE apresentou melhor desempenho cognitivo, quando comparado ao GC, após a intervenção. Conclusões: Podem ocorrer ganhos cognitivos associados a uma intervenção psicoeducativa para idosos hipertensos.

Palavras-chave: Idosos, cognição, treino cognitivo, hipertensão, psicoeducação.
\end{abstract}

\begin{abstract}
Objectives: To evaluate the possibility of combining cognitive training to an educational intervention composed by eight sessions about hypertension for a better management of the disease among the elderly. Methods: 64 older adults who reported having hypertension, divided into experimental group $(\mathrm{EG}, n=35)$ and control group $(\mathrm{CG}, n=29)$ participated in the study. Control participants received training after the post-test. The protocol contained socio-demographic and clinical data, Mini-Mental State Examination (MMSE), Geriatric Depression Scale (GDS), Rivermead Behavioral Memory Test (RBMT), Verbal Fluency Animal Category (VF) and Short Cognitive Test (SKT). Results: The EG showed better cognitive performance when compared with the CG, at post-test. Conclusion: Cognitive gains may occur after psychoeducational interventions for older adults with hypertension.

Keywords: Elderly people, cognition, cognitive training, hypertension, psychoeducation.
\end{abstract}

A hipertensão arterial tem sido associada a declínio em habilidades cognitivas ao longo do envelhecimento. Em indivíduos idosos, estudos têm demonstrado desempenho cognitivo mais baixo entre portadores de hipertensão, quando estes são comparados aos normotensos (Bortolotto, 1999). Autores ressaltam que as alterações cognitivas mais frequentes em indivíduos idosos hipertensos são disfunção executiva e problemas em atenção (Di Nucci, Coimbra, Neri, \& Yassuda, 2010).

Outros estudos documentam que as demências originadas por doenças vasculares apresentam uma taxa de prevalência que pode chegar a $30 \%$ entre as demências,

\footnotetext{
* Endereço para correspondência: Escola de Artes, Ciências e Humanidades, Universidade de São Paulo, Av. Arlindo Bettio, 1000, sala 322J, Ermelino Matarazzo, São Paulo, SP, Brasil 03828-000. E-mail: yassuda@usp.br Agradecimentos/Financiamentos: Esta pesquisa foi resultado de uma bolsa de iniciação cientifica realizada com o apoio financeiro da Fundação de Amparo à Pesquisa do Estado de São Paulo (FAPESP), sob a responsabilidade dos dois autores, projeto no. 2008/11918-3.
}

e que esse tipo de demência pode ser evitado ou postergado controlando-se os fatores de risco. A hipertensão arterial pode ser considerada como um fator preditivo de declínio cognitivo, visto que a hipertensão é um fator de risco para doenças responsáveis pela incapacidade e pela mortalidade por doenças vasculares (Hebert et al., 2004).

Estudos longitudinais têm demonstrado que a pressão arterial sistólica e a diastólica estão inversamente relacionadas com desempenho cognitivo em idosos (Cervilla, Prince, Joels, \& Mann, 2000; Elias, Elias, Robbins, \& Budge, 2004; Freitag et al., 2006; Waldstein, Giggey, Thayer, \& Zondermaman, 2005). Elias et al. (2004), por exemplo, examinaram as relações entre pressão arterial e declínio cognitivo em 529 indivíduos. Os participantes foram divididos em duas faixas etárias (18 a 46 anos e 47 a 83 anos) e foram testados ao longo de 20 anos. Os resultados mostraram que maiores níveis de pressão arterial sistólica e diastólica estiveram significativamente relacionados com o declínio em algumas funções cognitivas 
(visualização e habilidades fluidas), tanto no grupo mais jovem quanto no grupo mais velho. Ainda, os adultos jovens eram tão suscetíveis ao declínio cognitivo relacionado à hipertensão, quanto adultos mais velhos e idosos.

Estudos epidemiológicos brasileiros documentam que aproximadamente $7 \%$ dos idosos preenchem critérios para demência (Bottino et al., 2008) e 16\% apresentam algum grau de deterioração cognitiva e/ou funcional (Hototian et al., 2008). Estes estudos indicam que o declínio cognitivo, assim como as doenças crônicas constituem importantes problemas de saúde pública, considerando-se a dependência e o ônus familiar que acarretam. Estes dados reforçam a relevância das intervenções cognitivas. Ressalta-se que nas doenças crônicas, como a hipertensão, a adesão ao tratamento medicamentoso, a mudança no estilo de vida e a psicoeducação, podem ser importantes para prevenir o declínio da cognição, como sugerido por ensaios randomizados recentes (Peila, White, Masaki, Petrovick, \& Launer, 2006). As intervenções farmacológicas e não farmacológicas têm importância fundamental na preservação da boa qualidade de vida do indivíduo idoso hipertenso.

O tema treino cognitivo, aliado à ação psicoeducativa sobre doenças crônicas, é um tema pouco investigado no Brasil. Este estudo também se justifica pela necessidade de caracterizar o perfil cognitivo de idosos hipertensos, visto que alguns estudos (Freitag et al., 2006; Waldstein et al., 2005) sugerem que existem diferenças, quando são comparados aos idosos normotensos. No Brasil, Di Nucci et al. (2010) não encontraram diferenças significativas quanto compararam idosos hipertensos com alta adesão ao tratamento farmacológico a idosos normotensos.

\section{Método}

\section{Participantes}

Foram convidados para participar do estudo 120 idosos selecionados aleatoriamente de um grupo de 186 participantes de pesquisa anterior que se auto declararam como hipertensos. Destes, 38 aceitaram participar do estudo e compuseram o grupo experimental (GE). O grupo controle (GC) foi composto por idosos frequentadores de um centro de convivência da Zona Leste do Município de São Paulo, totalizando 30 idosos. Um participante foi excluído do GC, pois apresentava sintomas depressivos, e três participantes do GE foram excluídos, pois apresentaram pontuação abaixo da nota de corte, para sua escolaridade, no Mini-Exame do Estado Mental (MEEM). Estes participantes excluídos participaram da intervenção, no entanto, seus dados não foram utilizados nas análises. A amostra total foi de 64 idosos.

Os critérios de inclusão no estudo foram: relatar ter sido diagnosticado como hipertenso, ter 60 anos ou mais, não apresentar sintomas depressivos, não relatar diagnóstico de diabetes, não ter perfil cognitivo compatível com quadro demencial, e ter disponibilidade para participar das sessões de treino cognitivo.

\section{Instrumentos}

Foi aplicado um protocolo que incluiu variáveis sóciodemográficas e clínicas, o Mini- Exame do Estado Mental (MEEM; Brucki, Nitrini, Caramelli, Bertolucci, \& Okamoto, 2003); a Escala de Depressão Geriátrica (EDG; Almeida \& Almeida, 1999); Teste Comportamental de Memória de Rivermead (RBMT; Yassuda et al., 2010), Fluência Verbal categoria animais (TFV; Brucki \& Rocha, 2004; Caramelli, Carthery-Goulart, Porto, CharchatFichman, \& Nitrini, 2007) e Teste Cognitivo Breve (Short Cognitive Test [SKT]; Flaks, Forlenza, Pereira, Viola, \& Yassuda, 2009; Hartmut \& Hellmut, 1997). É importante ressaltar que duas versões diferentes do RBMT (respectivamente B e C) e do SKT (versões A e E) foram utilizadas no pré e no pós-teste, para minimizar o efeito de retestagem.

O questionário sociodemográfico e de dados clínicos incluiu idade, renda, anos de escolaridade, estado civil, estado geral de saúde. A pressão sistólica e diastólica dos participantes foi aferida cinco vezes (três aferições na postura sentada e duas aferições em pé) por meio de aparelho digital, colocado no pulso esquerdo do participante. Foram seguidas os procedimentos de estudo anterior (Soares, 2010). Os participantes foram questionados também se haviam recebido o diagnóstico de diabetes, pois idosos diabéticos não foram incluídos nesta pesquisa. Não foi realizado um levantamento abrangente de outras doenças que os participantes poderiam apresentar.

Em relação às variáveis cognitivas, utilizou-se: o MEEM que é um instrumento de rastreio cognitivo composto por questões agrupadas em sete categorias, cada uma delas planejada com o objetivo de avaliar funções cognitivas específicas. São elas: a orientação temporal e espacial, registro de três palavras, atenção e cálculo, evocação das três palavras registradas, linguagem e praxia visuo-construtiva. Seu escore varia de 0 a 30 pontos, sendo que valores mais baixos apontam para possível déficit cognitivo. Os seguintes pontos de corte foram adotados por nível de escolaridade: para analfabetos, 17 pontos; escolaridade de 1 a 4 anos, 22 pontos; de 5 a 8 anos, 24 pontos; acima de 8 anos, 26 pontos. Esses pontos de corte foram adaptados de Brucki et al. (2003), subtraindo um desvio padrão da média para a faixa de escolaridade, arredondando para baixo.

Para avaliar a presença de sintomas depressivos, foi aplicada a EDG que é um dos instrumentos mais utilizados para o rastreamento de depressão no idoso. No Brasil, Almeida e Almeida (1999) e Paradela, Lourenço e Veras, (2005) aplicaram a EDG-15 em 64 idosos de um ambulatório psiquiátrico, e ela mostrou-se confiável e válida para o rastreamento da depressão na população idosa brasileira.

O teste de FV é um instrumento simples, que consiste na nomeação do maior número possível de animais durante um minuto. Este teste avalia atenção, memória semântica, funções executivas e linguagem (Brucki \& Rocha, 2004; Caramelli et al., 2007). 
O SKT é aplicado para auxiliar na identificação e no acompanhamento do declínio cognitivo. $O$ teste consiste de nove subitens, sendo três destes relacionados à memória e seis relacionados à atenção. $\mathrm{O}$ domínio atenção varia de 0 a 18 pontos e o domínio memória varia de zero a nove pontos. O total do SKT varia de 0 a 27 pontos e menor pontuação sugere maior preservação cognitiva. Em média, o teste tem duração de 15 minutos e para analisálo é necessário conhecer a idade e o quociente de inteligência (QI) do participante (Hartmut \& Hellmeut, 1997). Quando o QI do paciente não é conhecido, os autores recomendam que o teste seja aferido considerando-se QI mediano. Este procedimento foi adotado neste estudo, visto que o QI não foi avaliado. O SKT encontra-se validado para a população idosa brasileira (Flaks et al., 2006; Flaks et al., 2009).

O Teste Comportamental de Memória de Rivermead (Wilson, Cockburn, Baddeley, \& Hiorns, 1989) foi utilizado com o intuito de caracterizar o perfil cognitivo dos participantes e como medida de desfecho para a intervenção cognitiva. É uma bateria ecológica que reproduz atividades da vida diária, como lembrar nomes, recados, rostos de pessoas, entre outras tarefas. Inclui 12 subitens e seu escore de triagem varia de zero a 12 pontos, e seu escore padronizado varia de zero a 24 pontos. Estudos brasileiros vêm demonstrando que a RBMT mantém suas propriedades psicométricas junto à população idosa brasileira (Yassuda et al., 2010). Todos os testes cognitivos foram usados como medida de eficácia da intervenção.

\section{Procedimentos}

Os idosos do GE foram avaliados no início do treinamento e após o treinamento de oito sessões. Os idosos do GC foram avaliados no mesmo período, mas o treinamento de oito sessões foi realizado após a coleta dos dados do pós teste. Durante o treino, os idosos receberam conteúdos educativos sobre envelhecimento cognitivo e sobre a hipertensão. As aulas sobre hipertensão foram baseadas na atenção farmacêutica, que visa a educação sobre o manejo da doença e principalmente a adesão ao tratamento medicamentoso. As estratégias cognitivas foram usadas como aliadas à promoção da alimentação e hábitos de vida mais saudáveis. Algumas tarefas cognitivas foram extraídas do livro Deu Branco (Alvarez, 2006). A programação completa das sessões encontra-se detalhada no Anexo A. As avaliações e os treinos foram realizados em duas organizações filantrópicas.

\section{Aspectos Éticos}

Este estudo foi aprovado pelo Comitê de Ética em Pesquisa do Instituto de Psicologia da Universidade de São Paulo, protocolo de número 005/08. Antes de iniciar a avaliação, cada participante da pesquisa assinou um Termo de Consentimento Livre e Esclarecido.

\section{Análises Estatísticas}

Para descrever o perfil da amostra segundo as variáveis em estudo, foram feitas tabelas de freqüência das variáveis categóricas, com valores de freqüência absoluta e percentual, e estatísticas descritivas das variáveis contínuas (idade, escores dos testes cognitivos), com valores de média, desvio padrão, valores mínimo, máximo, e mediana. Para comparação das variáveis categóricas entre os grupos foram utilizados o teste Qui-Quadrado, ou o teste exato de Fisher (na presença de valores esperados menores que 5). Ambos os testes comparam a proporção observada de uma determinada resposta com a proporção de respostas obtidas.

Foram realizadas análises com o teste de KolmogorovSmirnov para avaliar se as variáveis seguiam distribuição normal. Foi analisado se havia diferença estatística entre o GE e o GC para as variáveis sociodemográficas, cognitivas, no pré e pós teste, por meio do teste $t$ de Student para amostras independentes, na presença de distribuição normal. Quando não houve distribuição normal, foi utilizado o teste $U$ de Mann Whitney. Também foram realizadas comparações entre o pré e o pós teste para o GE e GC separadamente, por meio do teste $t$ de Student para amostras pareadas, e quando não houve distribuição normal, foi utilizado o teste de Wilcoxon para amostras pareadas.

Também foram calculados os deltas (escore do pós-teste menos o escore do pré-teste) para avaliar a evolução dos grupos GE e GC do pré para o pós-teste. Os deltas dos grupos GE e GC foram comparados Foi escolhido como nível de significância estatística $p<0,05$. Os dados foram digitados no Programa Epitada versão 3.1, e analisados pelo programa SPSS versão 17.0.

\section{Resultados}

A seguir, estão apresentados os resultados para as análises dos dados sociodemográficos e das variáveis cognitivas. Os participantes do GE e GC eram semelhantes quanto às variáveis sociodemográficas, com a exceção do estado civil, ocupação e renda (Tabela 1). O GE apresentava mais indivíduos casados, com maior renda e maior frequência de ocupação formal.

Os participantes do grupo GE e GC eram semelhantes em relação ao uso de medicamentos anti-hipertensivos. Apenas um participante do GC relatou não fazer uso de medicamentos. Em relação ao tempo médio de diagnóstico da hipertensão, no GE foi de 14,37 anos e no GC a média foi de 10, 25 anos, entretanto, esta diferença não atingiu significância estatística. Quanto à média das cinco aferições de pressão arterial, no pré-teste, não houve diferença significativa entre os grupos para a pressão arterial sistólica (PAS), entretanto, a pressão arterial diastólica (PAD) do GC foi significativamente mais elevada (Tabela 2). No pós teste, não foram identificadas alterações neste padrão, isto é, o CG apresentou PAD ligeiramente mais alta que o GE (dados não apresentados por se afastarem dos objetivos do estudo). 
Lima-Silva, T. B. \& Yassuda, M. S. (2012). Treino Cognitivo e Intervenção Psicoeducativa para Indivíduos Hipertensos: Efeitos na Cognição.

Tabela 1

Perfil Sócio-demográfico dos Participantes Estratificados em Grupo Controle e Experimental

\begin{tabular}{|c|c|c|c|c|c|}
\hline \multirow[t]{3}{*}{ Variável } & \multicolumn{5}{|c|}{ Grupos } \\
\hline & \multicolumn{2}{|c|}{ Experimental } & \multicolumn{2}{|c|}{ Controle } & \multirow[b]{2}{*}{$p$-valor } \\
\hline & $n$ & $\%$ & $n$ & $\%$ & \\
\hline \multicolumn{6}{|l|}{ Gênero } \\
\hline Feminino & 23 & 65,71 & 25 & 86,21 & \\
\hline Masculino & 12 & 34,29 & 4 & 13,79 & $0,083^{\mathrm{a}}$ \\
\hline \multicolumn{6}{|l|}{ Idade (em anos) } \\
\hline Média $(D P \pm)$ & 73,49 & 7,04 & 71,79 & 6,73 & $0,332^{\mathrm{b}}$ \\
\hline Mínimo-Máximo & \multicolumn{2}{|c|}{$60,00-92,00$} & \multicolumn{2}{|c|}{$61,00-90,00$} & \\
\hline \multicolumn{6}{|l|}{ Estado Civil } \\
\hline Casado(a) & 24 & 68,57 & 9 & 31,03 & \\
\hline Solteiro(a) & 0 & 0,00 & 5 & 17,24 & \\
\hline Viúvo(a) & 8 & 22,86 & 13 & 44,83 & \\
\hline Separado(a) & 3 & 8,57 & 1 & 3,45 & \\
\hline Divorciado(a) & 0 & 0,00 & 1 & 3,45 & $0,002^{\mathrm{c}}$ \\
\hline \multicolumn{6}{|l|}{ Escolaridade (anos) } \\
\hline Média $(D P \pm)$ & 5,63 & 1,78 & 5,24 & 2,40 & $0,110^{\mathrm{d}}$ \\
\hline Mínimo-Máximo & \multicolumn{2}{|c|}{$3,00-9,00$} & \multicolumn{2}{|c|}{$3,00-11,00$} & \\
\hline \multicolumn{6}{|l|}{ Trabalha } \\
\hline Sim & 16 & 45,71 & 4 & 13,79 & \\
\hline Não & 19 & 54,29 & 25 & 86,21 & $0,007^{\mathrm{a}}$ \\
\hline \multicolumn{6}{|l|}{ Renda Familiar } \\
\hline Média $(D P \pm)$ & 1403,09 & 365,64 & 1073,10 & 545,32 & $0,005^{\mathrm{b}}$ \\
\hline Mínimo-Máximo & \multicolumn{2}{|c|}{$785,00-2300,00$} & \multicolumn{2}{|c|}{$465,00-2100,00$} & \\
\hline
\end{tabular}

${ }^{\text {a }}$ Teste Qui-quadrado; ${ }^{\text {b Teste }} t$ para amostras independentes; ${ }^{\mathrm{c}}$ Teste Exato de Fischer; ${ }^{\mathrm{d}}$ Teste $U$ de Mann-Whitney.

Tabela 2

Análise Descritiva das Variáveis Clínicas Categóricas no Pré-teste

\begin{tabular}{|c|c|c|c|c|c|}
\hline \multirow[t]{3}{*}{ Variável } & \multicolumn{4}{|c|}{ Grupos } & \multirow[b]{3}{*}{$p$-valor } \\
\hline & \multicolumn{2}{|c|}{ Experimental } & \multicolumn{2}{|c|}{ Controle } & \\
\hline & $n$ & $\%$ & $N$ & $\%$ & \\
\hline \multicolumn{6}{|c|}{ Pratica exercícios físicos? } \\
\hline Sim & 21 & 60,00 & 22 & 75,86 & \\
\hline Não & 14 & 40,00 & 7 & 24,14 & $0,196^{\mathrm{a}}$ \\
\hline \multicolumn{6}{|c|}{ Se sim, quantas horas por semana? } \\
\hline Média $(D P \pm)$ & 2,86 & 1,35 & 2,73 & 1,45 & $0,672^{\mathrm{b}}$ \\
\hline Mínimo-Máximo & \multicolumn{2}{|c|}{$1,00-6,00$} & \multicolumn{2}{|c|}{$1,00-6,00$} & \\
\hline \multicolumn{6}{|c|}{ Toma remédio regularmente? } \\
\hline Sim & 35 & 100,00 & 28 & 96,55 & \\
\hline Não & 0 & 0,00 & 1 & 3,45 & $0,453^{\mathrm{a}}$ \\
\hline \multicolumn{6}{|c|}{ Mudanças na capacidade de enxergar? } \\
\hline Sim & 9 & 25,71 & 6 & 20,69 & \\
\hline Não & 26 & 74,29 & 23 & 79,31 & $0,770^{\mathrm{a}}$ \\
\hline \multicolumn{6}{|c|}{ Mudanças na capacidade de escutar? } \\
\hline Sim & 2 & 5,71 & 4 & 13,79 & \\
\hline Não & 33 & 94,29 & 25 & 86,21 & $0,397^{\mathrm{a}}$ \\
\hline
\end{tabular}


Alguma vez seu médico disse que você tem pressão alta?

Sim

Há quanto tempo recebeu o diagnóstico? (Anos)
Média $(D P \pm)$
Mínimo-Máximo

Toma medicamento para HAS?

Sim

Não

Frequência de uso do medicamento para HAS

Uma vez ao dia

Duas vezes ao dia

Três vezes ao dia

Esporadicamente

Não utiliza

Nas últimas semanas sua pressão esteve controlada?

$$
\text { Sim }
$$

Não

Pressão Arterial Sistólica mmHg (PAS)

Média $(D P \pm)$
Mínimo-Máximo

Pressão Arterial Diastólica mmHg (PAD)

Média $(D P \pm)$

Mínimo-Máximo
$35 \quad 100,00$

$14,37 \quad 8,582$

$1,00-35,00$

$35 \quad 100,00$

$0 \quad 0,00$

$19 \quad 54,29$

$10 \quad 28,57$

$1 \quad 2,86$

$5 \quad 14,29$

$0 \quad 0,00$

$32 \quad 91,43$

$3 \quad 8,57$

$141,50 \quad(10,94)$

$129,67-178,00$

$91,70 \quad(13,30)$

70,67-90,33
$29 \quad 100,00$

$1,000^{\mathrm{a}}$

$\begin{array}{cc}10,25 & 9,31 \quad 0,073^{c} \\ 1,00-43,00 & \end{array}$

2896,55

13,45

$0,453^{\mathrm{a}}$

$15 \quad 51,72$

$7 \quad 24,14$

$1 \quad 3,45$

$5 \quad 17,24$

$1 \quad 3,45$

$0,911^{\mathrm{d}}$

$26 \quad 89,66$

$3 \quad 10,34$

$1,000^{\mathrm{d}}$

$144,60 \quad(16,89)$

$122,33 \quad 207,33$

0,380

$100,77 \quad(10,87)$

0,005

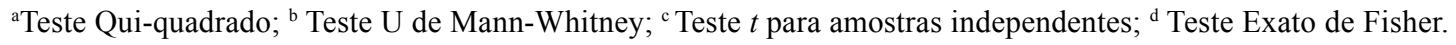

Tabela 3

Comparação do Desempenho Cognitivo do Grupo Experimental e Controle no Pré-teste

\begin{tabular}{llrrrrrrr}
\hline \multirow{2}{*}{ Variável } & \multicolumn{7}{c}{ Estatísticas Descritivas } \\
\cline { 2 - 8 } EDG & Grupo & $n$ & Média & $D P$ & Mínimo & Mediana & Máximo & $p$-valor \\
\hline \multirow{4}{*}{ MEEM } & Experimental & 35 & 4,89 & 0,83 & 2,00 & 5,00 & 6,00 & $0,007^{\mathrm{a}}$ \\
& Controle & 29 & 4,31 & 1,00 & 1,00 & 5,00 & 5,00 & \\
TFV & Experimental & 35 & 24,40 & 0,91 & 23,00 & 24,00 & 26,00 & $0,036^{\mathrm{a}}$ \\
& Controle & 29 & 25,00 & 1,13 & 23,00 & 25,00 & 28,00 & \\
\multirow{5}{*}{ SKT memória } & Experimental & 35 & 12,49 & 2,34 & 7,00 & 13,00 & 17,00 & $0,163^{\mathrm{b}}$ \\
& Controle & 29 & 13,34 & 2,51 & 10,00 & 12,00 & 19,00 & \\
SKT atenção & Experimental & 35 & 6,34 & 1,88 & 3,00 & 6,00 & 9,00 & $0,944^{\mathrm{b}}$ \\
& Controle & 29 & 6,38 & 2,24 & 1,00 & 7,00 & 9,00 & \\
SKT total & Experimental & 35 & 7,91 & 2,67 & 2,00 & 7,00 & 12,00 & $0,688^{\mathrm{b}}$ \\
& Controle & 29 & 7,62 & 3,14 & 2,00 & 8,00 & 16,00 & \\
\multirow{2}{*}{ RBMT padronizado } & Experimental & 35 & 14,26 & 4,10 & 5,00 & 13,00 & 21,00 & $0,809^{\mathrm{b}}$ \\
& Controle & 29 & 14,00 & 4,37 & 4,00 & 15,00 & 21,00 & \\
\multirow{2}{*}{ RBMT triagem } & Experimental & 35 & 12,26 & 2,19 & 7,00 & 12,00 & 16,00 & $0,133^{\mathrm{b}}$ \\
& Controle & 29 & 11,41 & 2,23 & 7,00 & 11,00 & 17,00 & \\
& Experimental & 35 & 3,46 & 1,20 & 1,00 & 3,00 & 6,00 & $0,190^{\mathrm{a}}$ \\
& Controle & 29 & 3,14 & 1,25 & 1,00 & 3,00 & 6,00 & \\
\hline
\end{tabular}

Nota. Mini-Exame do Estado Mental (MEEM); Escala de Depressão Geriátrica (EDG); Teste Comportamental de Memória de Rivermead RBMT; Fluência Verbal animais (TFV) e Short Cognitive Test (SKT).

${ }^{\text {a Teste }} U$ de Mann-Whitney; ${ }^{\text {b }}$ Teste $t$ de Student para amostras independentes. 
Lima-Silva, T. B. \& Yassuda, M. S. (2012). Treino Cognitivo e Intervenção Psicoeducativa para Indivíduos Hipertensos: Efeitos na Cognição.

Por meio da Tabela 3, pode-se verificar que no préteste os grupos apresentaram semelhanças em relação ao desempenho nas escalas cognitivas, com exceção da EDG e MEEM. O GE apresentou mais sintomas depressivos e menor desempenho no MEEM.

Na Tabela 4, verifica-se a comparação do desempenho cognitivo do GE e GC no pós-teste. Pode-se observar di- ferença significativa entre os dois grupos a favor GE, mostrando que as diferenças entre os dois grupos se acentuaram após a intervenção.

A Tabela 5 apresenta, separadamente por grupo, a comparação entre os escores no pré e pós teste. Os resultados indicam alteração positiva significativa no GE e manutenção dos escores no grupo controle.

Tabela 4

Comparação do Desempenho Cognitivo do Grupo Experimental e Controle no Pós-teste

\begin{tabular}{|c|c|c|c|c|c|c|c|c|}
\hline \multirow[b]{2}{*}{ Variável } & \multicolumn{7}{|c|}{ Estatísticas Descritivas } & \multirow[b]{2}{*}{$p$-valor } \\
\hline & Grupo & $n$ & Média & $D P$ & Mínimo & Mediana & Máximo & \\
\hline \multirow[t]{2}{*}{ EDG } & Experimental & 35 & 2,54 & 0,61 & 2,00 & 2,00 & 4,00 & \multirow[t]{2}{*}{$<0,001^{\mathrm{a}}$} \\
\hline & Controle & 29 & 4,52 & 0,74 & 3,00 & 5,00 & 5,00 & \\
\hline \multirow[t]{2}{*}{ MEEM } & Experimental & 35 & 27,09 & 0,82 & 25,00 & 27,00 & 28,00 & \multirow[t]{2}{*}{$<0,001^{\mathrm{a}}$} \\
\hline & Controle & 29 & 25,28 & 1,13 & 24,00 & 25,00 & 28,00 & \\
\hline \multirow[t]{2}{*}{ TFV } & Experimental & 35 & 16,29 & 1,60 & 13,00 & 16,00 & 19,00 & \multirow[t]{2}{*}{$<0,001$} \\
\hline & Controle & 29 & 13,52 & 1,92 & 11,00 & 14,00 & 18,00 & \\
\hline \multirow[t]{2}{*}{ SKT memória } & Experimental & 35 & 2,77 & 1,09 & 2,00 & 3,00 & 8,00 & \multirow[t]{2}{*}{$<0,001^{\mathrm{a}}$} \\
\hline & Controle & 29 & 6,66 & 2,07 & 2,00 & 7,00 & 9,00 & \\
\hline \multirow[t]{2}{*}{ SKT atenção } & Experimental & 35 & 2,54 & 1,52 & 0,00 & 2,00 & 9,00 & \multirow{2}{*}{$<0,001$} \\
\hline & Controle & 29 & 7,34 & 2,81 & 2,00 & 8,00 & 12,00 & \\
\hline \multirow[t]{2}{*}{ SKT total } & Experimental & 35 & 5,31 & 2,41 & 2,00 & 5,00 & 17,00 & \multirow[t]{2}{*}{$<0,001$} \\
\hline & Controle & 29 & 14,00 & 4,57 & 5,00 & 15,00 & 21,00 & \\
\hline \multirow[t]{2}{*}{ RBMT padronizado } & Experimental & 35 & 18,26 & 1,99 & 14,00 & 18,00 & 22,00 & \multirow[t]{2}{*}{$<0,001^{\mathrm{b}}$} \\
\hline & Controle & 29 & 11,24 & 2,47 & 7,00 & 11,00 & 16,00 & \\
\hline \multirow[t]{2}{*}{ RBMT triagem } & Experimental & 35 & 7,43 & 1,38 & 5,00 & 7,00 & 10,00 & \multirow[t]{2}{*}{$<0,001^{\mathrm{b}}$} \\
\hline & Controle & 29 & 3,14 & 1,53 & 1,00 & 3,00 & 7,00 & \\
\hline
\end{tabular}

Nota. Mini-Exame do Estado Mental (MEEM); Escala de Depressão Geriátrica (EDG); Teste Comportamental de Memória de Rivermead RBMT; Fluência Verbal animais (TFV) e Short Cognitive Test (SKT).

${ }^{\mathrm{a}}$ Teste $U$ de Mann-Whitney; ${ }^{\mathrm{b}}$ Teste $t$ de Student para amostras independentes.

Tabela 5

Comparação das Escalas Cognitivas no Pré e no Pós-teste entre os Grupos Experimental e Controle

\begin{tabular}{|c|c|c|c|c|c|c|c|c|}
\hline \multirow[b]{2}{*}{ Variável } & \multicolumn{7}{|c|}{ Estatísticas Descritivas } & \multirow[b]{2}{*}{$p$-valor } \\
\hline & Grupo & Tempo & Média & $D P$ & Mínimo & Mediana & Máximo & \\
\hline \multirow[t]{4}{*}{ EDG } & Experimental & Pré-teste & 4,89 & 0,83 & 2,00 & 5,00 & 6,00 & $<0,001^{\mathrm{a}}$ \\
\hline & & Pós-teste & 2,54 & 0,61 & 2,00 & 2,00 & 4,00 & \\
\hline & Controle & Pré-teste & 4,31 & 1,00 & 1,00 & 5,00 & 5,00 & $0,478^{\mathrm{a}}$ \\
\hline & & Pós-teste & 4,52 & 0,74 & 3,00 & 5,00 & 5,00 & \\
\hline \multirow{4}{*}{ MEEM } & Experimental & Pré-teste & 24,40 & 0,91 & 23,00 & 24,00 & 26,00 & $<0,001^{\mathrm{a}}$ \\
\hline & & Pós-teste & 27,09 & 0,82 & 25,00 & 27,00 & 28,00 & \\
\hline & Controle & Pré-teste & 25,00 & 1,13 & 23,00 & 25,00 & 28,00 & $0,546^{\mathrm{a}}$ \\
\hline & & Pós-teste & 25,28 & 1,13 & 24,00 & 25,00 & 28,00 & \\
\hline \multirow[t]{4}{*}{ TFV } & Experimental & Pré-teste & 12,49 & 2,34 & 7,00 & 13,00 & 17,00 & $<0,001^{\mathrm{b}}$ \\
\hline & & Pós-teste & 16,29 & 1,60 & 13,00 & 16,00 & 19,00 & \\
\hline & Controle & Pré-teste & 13,34 & 2,51 & 10,00 & 12,00 & 19,00 & $0,723^{\mathrm{b}}$ \\
\hline & & Pós-teste & 13,52 & 1,92 & 11,00 & 14,00 & 18,00 & \\
\hline
\end{tabular}


Psicologia: Reflexão e Crítica, 25(1), 30-40.

\begin{tabular}{|c|c|c|c|c|c|c|c|c|}
\hline \multirow[t]{4}{*}{ SKT memória } & \multirow[t]{2}{*}{ Experimental } & Pré-teste & 6,34 & 1,88 & 3,00 & 6,00 & 9,00 & \multirow[t]{2}{*}{$<0,001^{\mathrm{a}}$} \\
\hline & & Pós-teste & 2,77 & 1,09 & 2,00 & 3,00 & 8,00 & \\
\hline & \multirow[t]{2}{*}{ Controle } & Pré-teste & 6,38 & 2,24 & 1,00 & 7,00 & 9,00 & \multirow[t]{2}{*}{$0,728^{\mathrm{a}}$} \\
\hline & & Pós-teste & 6,66 & 2,07 & 2,00 & 7,00 & 9,00 & \\
\hline \multirow{4}{*}{ SKT atenção } & \multirow[t]{2}{*}{ Experimental } & Pré-teste & 7,91 & 2,67 & 2,00 & 7,00 & 12,00 & \multirow[t]{2}{*}{$<0,001^{\mathrm{a}}$} \\
\hline & & Pós-teste & 2,54 & 1,52 & 0,00 & 2,00 & 9,00 & \\
\hline & \multirow[t]{2}{*}{ Controle } & Pré-teste & 7,62 & 3,14 & 2,00 & 8,00 & 16,00 & \multirow[t]{2}{*}{$0,899^{\mathrm{a}}$} \\
\hline & & Pós-teste & 7,34 & 2,81 & 2,00 & 8,00 & 12,00 & \\
\hline \multirow[t]{4}{*}{ SKT total } & \multirow[t]{2}{*}{ Experimental } & Pré-teste & 14,26 & 4,10 & 5,00 & 13,00 & 21,00 & \multirow[t]{2}{*}{$<0,001^{\mathrm{a}}$} \\
\hline & & Pós-teste & 5,31 & 2,41 & 2,00 & 5,00 & 17,00 & \\
\hline & \multirow[t]{2}{*}{ Controle } & Pré-teste & 14,00 & 4,37 & 4,00 & 15,00 & 21,00 & \multirow{2}{*}{$0,747^{\mathrm{a}}$} \\
\hline & & Pós-teste & 14,00 & 4,57 & 5,00 & 15,00 & 21,00 & \\
\hline \multirow[t]{4}{*}{ RBMT padronizado } & \multirow[t]{2}{*}{ Experimental } & Pré-teste & 12,26 & 2,19 & 7,00 & 12,00 & 16,00 & \multirow[t]{2}{*}{$<0,001^{\mathrm{b}}$} \\
\hline & & Pós-teste & 18,26 & 1,99 & 14,00 & 18,00 & 22,00 & \\
\hline & \multirow[t]{2}{*}{ Controle } & Pré-teste & 11,41 & 2,23 & 7,00 & 11,00 & 17,00 & \multirow[t]{2}{*}{$0,701^{b}$} \\
\hline & & Pós-teste & 11,24 & 2,47 & 7,00 & 11,00 & 16,00 & \\
\hline \multirow[t]{4}{*}{ RBMT triagem } & \multirow[t]{2}{*}{ Experimental } & Pré-teste & 3,46 & 1,20 & 1,00 & 3,00 & 6,00 & \multirow[t]{2}{*}{$<0,001^{\mathrm{a}}$} \\
\hline & & Pós-teste & 7,43 & 1,38 & 5,00 & 7,00 & 10,00 & \\
\hline & \multirow[t]{2}{*}{ Controle } & Pré-teste & 3,14 & 1,25 & 1,00 & 3,00 & 6,00 & \multirow[t]{2}{*}{$0,935^{\mathrm{a}}$} \\
\hline & & Pós-teste & 3,14 & 1,53 & 1,00 & 3,00 & 7,00 & \\
\hline
\end{tabular}

Nota. Mini-Exame do Estado Mental (MEEM); Escala de Depressão Geriátrica (EDG); Teste Comportamental de Memória de Rivermead RBMT; Fluência Verbal animais (TFV) e Short Cognitive Test (SKT).

${ }^{\text {a }}$ Teste de Wilcoxon; ${ }^{\text {b }}$ teste $t$ de Student para amostras pareadas.

Tabela 6

Deltas (escores do pós menos o pré-teste) do Desempenho dos Participantes

\begin{tabular}{|c|c|c|c|c|c|c|c|c|}
\hline \multirow[b]{2}{*}{ Variável } & \multicolumn{7}{|c|}{ Estatísticas Descritivas } & \multirow[b]{2}{*}{$p$-valor } \\
\hline & Grupo & $n$ & Média & $D P$ & Mínimo & Mediana & Máximo & \\
\hline \multirow[t]{2}{*}{ EDG } & Experimental & 35 & $-2,34$ & 1,11 & $-4,0$ & $-2,00$ & 1,00 & \multirow[t]{2}{*}{$<0,001^{\mathrm{a}}$} \\
\hline & Controle & 29 & 0,21 & 1,24 & $-2,00$ & 0,00 & 4,00 & \\
\hline \multirow[t]{2}{*}{ MEEM } & Experimental & 35 & 2,69 & 1,08 & 0,0 & 3,00 & 5,00 & \multirow[t]{2}{*}{$<0,001^{\mathrm{a}}$} \\
\hline & Controle & 29 & 0,28 & 1,65 & $-3,00$ & 0,00 & 5,00 & \\
\hline \multirow[t]{2}{*}{ TFV } & Experimental & 35 & 3,80 & 2,10 & $-3,0$ & 4,00 & 7,00 & \multirow[t]{2}{*}{$<0,001^{\mathrm{a}}$} \\
\hline & Controle & 29 & 0,17 & 2,59 & $-7,00$ & 0,00 & 4,00 & \\
\hline \multirow[t]{2}{*}{ SKT memória } & Experimental & 35 & $-3,57$ & 1,84 & $-7,0$ & $-4,00$ & 1,00 & \multirow[t]{2}{*}{$<0,001^{\mathrm{a}}$} \\
\hline & Controle & 29 & 0,28 & 1,81 & $-2,00$ & 0,00 & 6,00 & \\
\hline \multirow[t]{2}{*}{ SKT atenção } & Experimental & 35 & $-5,37$ & 2,86 & $-11,0$ & $-5,00$ & 0,00 & \multirow[t]{2}{*}{$<0,001^{\mathrm{a}}$} \\
\hline & Controle & 29 & $-0,28$ & 3,02 & $-12,00$ & 0,00 & 4,00 & \\
\hline \multirow[t]{2}{*}{ SKT total } & Experimental & 35 & $-8,94$ & 4,12 & $-16,0$ & $-9,00$ & 1,00 & \multirow[t]{2}{*}{$<0,001^{\mathrm{a}}$} \\
\hline & Controle & 29 & 0,00 & 2,80 & $-6,00$ & 0,00 & 4,00 & \\
\hline \multirow[t]{2}{*}{ RBMT padronizado } & Experimental & 35 & 6,00 & 3,21 & 0,0 & 6,00 & 12,00 & \multirow[t]{2}{*}{$<0,001^{\mathrm{a}}$} \\
\hline & Controle & 29 & $-0,17$ & 2,39 & $-4,00$ & 0,00 & 4,00 & \\
\hline \multirow[t]{2}{*}{ RBMT triagem } & Experimental & 35 & 3,97 & 2,04 & 0,0 & 4,00 & 8,00 & \multirow[t]{2}{*}{$<0,001^{\mathrm{a}}$} \\
\hline & Controle & 29 & $-0,00$ & 1,73 & $-4,00$ & 0,00 & 4,00 & \\
\hline
\end{tabular}

Nota. Mini-Exame do Estado Mental (MEEM); Escala de Depressão Geriátrica (EDG); Questionário sobre Conhecimento sobre Hipertensão; Teste Comportamental de Memória de Rivermead RBMT; Fluência Verbal animais (TFV) e Short Cognitive Test (SKT).

a Teste $t$ de Student para amostras independentes. 
A análise dos deltas revelou diferença significativa entre os dois grupos para as escalas cognitivas, sugerindo que houve maior ganho entre os dois momentos de avaliação para o GE (Tabela 6).

\section{Discussão}

Estudos recentes vêm demonstrando que o treino cognitivo pode ser uma intervenção eficaz para idosos saudáveis e com diagnóstico de comprometimento cognitivo leve (Belleville, Chetrkow, \& Gauthier, 2007; Brum, Forlenza, \& Yassuda, 2009; Rapp, Brenes, \& Marsh, 2002). Entretanto, não foram localizados estudos de treino cognitivo em populações com doenças crônicas como a hipertensão, e sua capacidade de prevenir ou postergar a conversão para possíveis quadros demenciais.

Neste estudo, 64 idosos (35-GE e 29-GC), não acometidos por demência, previamente diagnosticados como portadores de hipertensão participaram de uma intervenção cognitiva de oito sessões. Foram detectadas alterações significativas no desempenho cognitivo dos participantes do GE em testes de atenção, memória, funções executivas, e diminuição de sintomas depressivos. O presente estudo apontou para a manutenção da plasticidade cognitiva entre idosos com hipertensão.

A diminuição de sintomas depressivos talvez seja explicada pelos relacionamentos sociais estabelecidos no grupo e pelo convívio com pessoas com a mesma situação de saúde. Também é possível que a oportunidade de realizar treino e melhorar o desempenho cognitivo tenha exercido impacto nos sintomas depressivos, como encontrado nos estudos de Brum et al. (2009), Olchik (2008) e Talassi et al. (2007). Entretanto, destaca-se que o aumento de conhecimentos sobre a HAS e sobre maneiras de tratar esta condição crônica pode ter gerado maior percepção de controle sobre a mesma e estar associado à observada diminuição nos sintomas depressivos.

Estudos sobre a plasticidade cognitiva revelam-se um campo fértil para a pesquisa básica, considerando que gera informações essenciais a respeito do envelhecimento humano. Ao mesmo tempo, estes estudos têm implicações importantes para a prática clínica do profissional gerontólogo, pois a otimização da memória está rela-cionada à saúde, à autonomia e independência do idoso. Ramos (2003) identificou em estudo epidemiológico que dependência funcional e declínio cognitivo associaram-se à mortalidade, e destacou que estes fatores são pas-síveis de intervenção. Adicionalmente, o treino aliado à psicoeducação pode resultar em melhor adesão ao tratamento da hipertensão proposto pelos profissionais de saúde. Pode-se supor também que a melhora cognitiva proporcionada pelo treino auxilie no controle da doença, pois esquecimentos e desatenção podem se associar ao uso irregular das medicações, dieta inadequada, cancelamento de consultas, entre outros pontos desfavoráveis.
O perfil de idosos avaliados pode ter contribuído para a eficácia do treino, visto que muitos estavam vinculados a centros de convivência, e a escolaridade da amostra era elevada, quando comparada à população idosa brasileira. Destaca-se a forte adesão à proposta da intervenção, visto que não houve evasão. Os participantes relataram apreciar a oportunidade de participar de uma atividade diferente das usualmente oferecidas. Pode-se supor também que o treino cognitivo tenha se tornado mais atraente aos participantes, pois tinha como objetivo a melhora da condição crônica e da cognição simultaneamente.

Em suma, a área de investigação que relaciona desempenho cognitivo e doenças crônicas está em expansão e abre espaço para a discussão sobre a promoção da saúde da população de diferentes faixas etárias. Limitações do presente estudo incluem uma amostra relativamente pequena, e um número de sessões limitado frente à complexidade dos temas abordados. Outras limitações metodológicas incluem o fato dos participantes não terem sido distribuídos randomicamente, e não ter sido realizado um levantamento de outras doenças associadas à hipertensão, visto que estas poderiam contribuir para as diferenças entre os grupos e no impacto do treino. Para estudos futuros sugere-se a replicação da metodologia do presente estudo, porém, com uma intervenção mais prolongada e com seguimento longitudinal dos participantes, para detectar a possível manutenção dos resultados.

\section{Referências}

Almeida, O. P., \& Almeida, S. A. (1999). Short versions of the geriatric depression scale: A study of their validity for the diagnosis of a major depressive episode according to ICD10 and DSM-IV. International Journal of Geriatric Psychiatry, 14(10), 858-865.

Alvarez, A. (2006). Deu branco (2. ed.). São Paulo, SP: Best Seller.

Belleville, S., Chetrkow, H., \& Gauthier, S. (2007). Working memory and control of attention in person with Alzheimer's disease and mild cognitive impairment. Neuropsychology, 21, 456-469.

Bortolotto, L. A. (1999). Distúrbios cognitivos no idoso hipertenso. Revista Brasileira de Hipertensão, 6(4), 388-393.

Bottino, C. M., Azevedo, D., Tatsch, M., Hotatian, S. R., Moscoso, M. A., Folquito, J., et al. (2008). Estimate of dementia prevalence in a community sample from São Paulo. Dementia Geriatric Cognitive Disorders, 26(4), 291-299.

Brucki, S. M. D., Nitrini, R., Caramelli, P., Bertolucci, P. H. F., \& Okamoto, I. H. (2003). Sugestões para o uso do mini-exame do estado mental no Brasil. Arquivos de Neuropsiquiatria, 61(3-B), 777-781.

Brucki, S. M. D., \& Rocha, M. S. G. (2004). Category fluency verbal: Effectes of age, gender and education on total scores, clustering and switching in Brazilian Portuguese-speaking subjects. Brazilian Journal of Medical and Biological Research, 37(12), 1771-1777.

Brum, P., Forlenza, O. V., \& Yassuda, M. S. (2009). Cogntive training in mild cognitive impairment. Dementia \& Neuropsychologia, 3(2), 127-131. 
Caramelli, P., Carthery-Goulart, M. T., Porto, C. S., CharchatFichman, H., \& Nitrini, R. (2007). Category fluency as a screening test for Alzheimer Disease in illiterate and and literate patients. Alzheimer Disease and Associated Disorders, 21(1), 65-67.

Cervilla, J. A., Prince, M., Joels, S., \& Mann, A. (2000). Does depression predict cognitive outcome 9 to 12 years later? Evidence from a prospective study of elderly hypertensives. Psychological Medicine, 30, 1017-1023.

Di Nucci, F. R. C. F., Coimbra, A. M. V., Neri, A. L., \& Yassuda, M. S. (2010). Ausência de relação entre hipertensão arterial sistêmica e desempenho cognitivo em idosos de uma comunidade. Revista de Psiquiatria Clínica, 37, 52-56.

Elias, P. K., Elias, M. F., Robbins, M. A., \& Budge, M. M. (2004). Blood pressure-related cognitive decline: Does age make a difference? Hypertension, 44, 631-636.

Flaks, M. K., Yassuda, M. S., Regina, A. C. B., Cid, C. G., Camargo, C. H. P., Gattaz, W. F., et al. (2006). The Short Test (SKT) a transcultural test for early detection and discrimination of dementia: A preliminary study in Brazil. International Psychogeriatrics, 18(1), 121-133.

Flaks, M. K., Forlenza, O. V., Pereira, F. S., Viola, L. F., \& Yassuda, M. S. (2009). Short cognitive performance test: diagnostic accuracy and education bias in older Brazilian adults. Archives of Clinical Neuropsychology, 24, 301-306.

Freitag, M. H., Peila, R., Masaki, K., Petrovich, H., Ross, G. W., White, L. R., et al. (2006). Midlife pulse pressure ans incidence of dementia. The Honolulu-Asia Aging Study. Stroke, 37, 33-37.

Hartmut, L., \& Hellmut, E. (1997). The SKT- A Short Cognitive Performance Test for Assessing Deficits of Memory and Attention. International Psychogeriatrics, 9, 115-121.

Hebert, L. E., Scherr, P. A., Bennett, D. A., Bienias, J. L., Wilson, R. S., \& Morris, M. C. (2004). Blood pressure and latelife cognitive function change. A biracial longitudinal population study. Neurology, 62, 2021-2024.

Hototian, S. R., Lopes, M. A., Azevedo, D., Tatsch, M., Bazarella, M. C., Bustamante, S. E., et al. (2008). Prevalence of cognitive and functional impairment in a community sample from São Paulo. Dementia and Geriatric Cognitive Disorders, 25(2), 135-143.

Olchik, M. R. (2008). Treino de memória: Um novo aprender no envelhecimento. (Tese de Doutorado não-publicada). Universidade Federal do Rio Grande do Sul, Porto Alegre, RS.

Paradela, E. M. P., Lourenço, R. A., \& Veras, R. (2005). Validação da escala de depressão geriátrica em um ambulatório geral. Revista de Saúde Pública, 39(6), 918-923.

Peila, R., White, L. R., Masaki, K., Petrovick, H., \& Launer, L. J. (2006). Reducing the risk of dementia: efficacy of longterm treatment of hypertension. Stroke, 37, 1165-1670.

Ramos, L. R. (2003). Fatores determinantes do envelhecimento saudável em idosos residentes em centro urbano: Projeto Epidoso, São Paulo. Cadernos de Saúde Pública, 19, 793797.

Rapp, S., Brenes, G., \& Marsh, A. P. (2002). Memory enhancement training for older adults with mild cognitive impairment: A preliminary study. Aging and Mental Health, 6, 5-11.

Soares, L. M. (2010). Relação entre cognição, hipertensão e diabetes em homens e mulheres idosos recrutados na comunidade. Dados do Projeto Fibra. (Dissertação de Mestrado não-publicada). Faculdade de Ciências Médicas, Universidade Estadual de Campinas, SP.
Talassi, E., Guerrreschi, M., Feriani, M., Fedi, V., Bianchetti, A., \& Trabucchi, M. (2007). Effectiveness of a cognitive rehabilitation program in mild dementia (MD) and mild cognitive impairment (MCI): A case control study. Archives of Gerontology and Geriatrics, 1(Suppl.), 391-399.

Waldstein, S. R., Giggey, P. P., Thayer, J. F., \& Zondermaman, A. B. (2005). Nonlinear relations of blood pressure to cognitive function: The Baltimore longitudinal Study of aging. Hypertension, 45, 374-379.

Wilson, B. A., Cockburn, J. M., Baddeley, A. D., \& Hiorns, R. (1989). The development and validation of a test battery for detecting and monitoring everyday memory problems. Journal of Clinical and Experimental Neuropsychology, 11(6), 855-870.

Yassuda, M. S., Flaks, M. K., Viola, L. F., Pereira, F. S., Memória, C. M., Nunes, P. V., et al. (2010). Psychometric characteristics of the Rivermead Behavioural Memory Test (RBMT) as an early detection instrument for dementia and mild cognitive impairment in Brazil. International Psychogeriatrics, 22(6), 1003-1111. 
Lima-Silva, T. B. \& Yassuda, M. S. (2012). Treino Cognitivo e Intervenção Psicoeducativa para Indivíduos Hipertensos: Efeitos na Cognição.

\section{$\operatorname{Anexo} A$}

\section{Programação da Intervenção}

$1^{\circ}$ Sessão. Apresentação da instrutora e dos participantes. Realização de sondagem com os participantes, para saber o que sabiam e o que gostariam de saber sobre hipertensão. Introdução geral sobre envelhecimento saudável e patológico (doenças crônicas, em particular a hipertensão), e introdução geral sobre memória no envelhecimento e seus subsistemas. Realização de exercício de atenção e tarefa lúdica: os participantes recebiam um poema, intitulado Motivo de Cecília Meirelles, e em seguida deveriam ler e procurar onde estavam algumas das palavras mencionadas pela instrutora.

$2^{\circ}$ Sessão. Aula psicoeducativa: "O que é hipertensão?". Breve explicação sobre a importância da atenção para o bom funcionamento da memória e sobre a relação hipertensão arterial não-controlada e dificuldades em atenção. Exercício de memória auditiva: os participantes tiveram que contar mentalmente quantas vezes uma palavra era mencionada em um pequeno texto sobre saúde lido pela instrutora. Exercício de atenção dominó fonológico: um participante falava uma palavra, por exemplo, dieta, e o seguinte deveria dizer uma palavra com a sílaba final da palavra do participante anterior (tabaco). Tarefa de funções executivas (tarefa extraída do Livro Deu Branco, p. 93): os participantes deveriam colocar em ordem alfabética palavras lidas pela instrutora, como xaxim, casa e beterraba (beterraba, casa e xaxim).

$3^{\circ}$ Sessão. Aula psicoeducativa sobre hipertensão e a influência do sal. Introdução sobre o uso de estratégias de memória para o melhor desempenho cognitivo e melhor manejo da doença. Foram apresentados alguns exemplos do uso de imagens mentais, associações, e categorização. Exercício de atenção visual: os participantes tiveram que achar todos os quadrados em uma folha repleta de pequenas figuras geométricas. Receberam imagens de pessoas realizando atividades físicas e foram questionados sobre as figuras. Valores nutricionais dos alimentos: explicou-se a importância do consumo adequado de sódio. Foram utilizadas embalagens de alimentos industrializados. Atividade de funções executivas: foram realizados cálculos de matemática básica para que os participantes soubessem se estavam ultrapassando valores necessários diários de sódio. Um dia antes do próximo encontro cada participante tinha que anotar os nomes dos alimentos que consumia e a quantidade, para que fosse possível calcular as quantidades de sódio consumidas e sua relação com o valor diário (\%) que aquela quantidade representava.

$4^{\circ}$ Sessão. Aula psicoeducativa: hipertensão a influencia do fumo e do estresse. Revisão da estratégia de categorização e exercício com palavras pertencentes a três categorias semânticas diferentes. Os participantes receberam uma lista com dez itens de alimentos categorizáveis em saudáveis e não saudáveis para hipertensão. Os idosos agruparam esses itens de acordo com a categoria a que pertenciam. Discussão sobre a importância da alimentação para a hipertensão. Exercício de memória operacional do livro Deu Branco: os participantes deveriam colocar palavras ditas pela instrutora (adulto, bebê, adolescente) na sequência temporal correta (bebê, adolescente, adulto).

$5^{\circ}$ Sessão. Aula psicoeducativa: hipertensão e a importância do exercício físico e da mudança no estilo de vida (com auxílio teórico de apostila educativa). Exercício de atenção auditiva: os participantes ouviram uma lista de seis manchetes de jornal recentes sobre hábitos de vida saudáveis, extraídas de uma revista sobre saúde, e a cada uma tiveram que decidir levantando a mão direita ou esquerda se era uma boa ou má notícia, respectivamente. Também deveriam recordar-se da última palavra de cada notícia. Atividade de memória operacional: os participantes recebiam cartões orientando-os a tomar medicamento anti-hipertensivo em um horário, como em: "o senhor deverá tomar este medicamento duas vezes ao dia de doze em doze horas...". Perguntou-se a cada participante, com diferentes horários "se o senhor tomá-lo às 8:00 horas, a que horas terá que tomar o medicamento novamente?". Atividade de fluência verbal e memorização: "eu vou ao médico e vou levar...", cada pessoa, antes de falar o objeto que levaria, deveria falar também o objeto que as pessoas anteriores falaram.

$6^{\circ}$ Sessão. Aula teórica sobre medicamentos antihipertensivos, com apoio de um folheto educativo, e momento de esclarecimentos de dúvidas sobre os fármacos, como seguir um tratamento adequado, a importância de auxílios externos (caixinhas de remédios, alarmes). Tarefa de atenção auditiva visual com a música Beatriz de Chico Buarque: os participantes deveriam detectar dentre uma lista de palavras escritas em uma folha quais apareciam na música. Atividade de categorização de alimentos saudáveis e não saudáveis com embalagens de supermercado.

$7^{\circ}$ Sessão. Aula psicoeducativa com ênfase na hipertensão e a importância da aferição da pressão. Explicação teórica sobre memória operacional. Atividade de atenção auditiva com manchetes de jornal. Tarefa de atenção e memória episódica: o participante recebia uma folha com uma imagem a ser estudada e posteriormente deveria responder algumas perguntas sem olhar a figura. Realização da atividade STOP: exercício de fluência verbal com restrição categórica e fonológica, os participantes tinham que pensar em itens como frutas, flores, animais, nomes próprios com uma determinada letra. Tarefa de funções executivas extraídas do Livro Deu Branco, p. 91: 
a instrutora lia palavras desorganizadas que deveriam ser organizadas de modo a formar frases.

$8^{\circ}$ Sessão. Revisão da parte teórica e último dia de atividades: Aula psicoeducativa com orientações focando a adesão ao tratamento antihipertensivo. Realização de atividade de fluência verbal: participantes deveriam dizer itens que eram encontrados em fazendas. Repetição do exercício com itens encontrados na cozinha. Exercício de categorização com 15 palavras que pertenciam a três categorias diferentes. Tarefa de ordenação temporal: os participantes recebiam sequências de palavras e deveriam dizer o que acontece primeiro, por exemplo, dormir, acordar, sonhar (dormir, sonhar, acordar). Festa de encerramento. 